\title{
ON PARRONDO'S PARADOX: HOW TO CONSTRUCT UNFAIR GAMES BY COMPOSING FAIR GAMES
}

\author{
E. S. KEY ${ }^{\varpi}$, M. M. KŁOSEK ${ }^{2}$ and D. ABBOTT ${ }^{3}$
}

(Received 26 October, 2000; revised 5 May, 2006)

\begin{abstract}
We construct games of chance from simpler games of chance. We show that it may happen that the simpler games of chance are fair or unfavourable to a player and yet the new combined game is favourable-this is a counter-intuitive phenomenon known as Parrondo's paradox. We observe that all of the games in question are random walks in periodic environments (RWPE) when viewed on the proper time scale. Consequently, we use RWPE techniques to derive conditions under which Parrondo's paradox occurs.
\end{abstract}

2000 Mathematics subject classification: primary $60 \mathrm{~J} 10$; secondary $60 \mathrm{~K} 37,82 \mathrm{C} 41$.

Keywords and phrases: random walk in a periodic environment, random transport, random games, Parrondo's paradox.

\section{Introduction}

Parrondian strategies are where losing games can cooperate to win [6]. The original example of Parrondo's games consist of two coin tossing games. Game A consists of Coin 1 biased to lose. Game B consists of two coins-Coin 2 with losing bias and Coin 3 with winning bias-but a state-dependent rule is chosen to favour the losing Coin 2. Hence both games A and B are losing games. However when A and $B$ are alternated in a deterministic or even random manner, surprisingly the player has a winning expectation. This effect has been interpreted in terms of a

\footnotetext{
'Department of Mathematical Sciences, University of Wisconsin Milwaukee, Milwaukee WI 53201, USA; e-mail: ericskey@uwm.edu.

${ }^{2}$ Department of Mathematical Sciences, University of Wisconsin Milwaukee, Milwaukee WI 53201, USA. Currently at the Center of Scientific Review, National Institutes of Health. This article was written in a personal capacity and does not necessarily represent the opinions or reflect the views of the National Institutes of Health, the Department of Health and Human Services, or the Federal Government. ${ }^{3}$ Centre for Biomedical Engineering (CBME), Department of Electrical \& Electronic Engineering, University of Adelaide, Adelaide SA 5005 Australia; e-mail: dabbott@eleceng,adelaide.edu.au. (C) Australian Mathematical Society 2006, Serial-fee code 1446-181 1/06
} 
discrete-time Brownian ratchet at length elsewhere [7], where conventional Brownian ratchets [2] have been the inspiration. An alternative view, which we call the Boston interpretation [15], recognises that although game B favours Coin 2 with losing bias, if the state-dependence is removed, game $B$ now favours the winning Coin 3-then when games $A$ and $B$ are mixed, game $A$ has the affect of randomisation or 'breakup' of game B's state-dependence, thus tilting favour towards Coin 3 with winning bias. This explanation was also independently deduced by J. Maynard Smith [19]. In addition to the ratchet interpretation and the Boston interpretation, this paper will examine another viewpoint by considering the process as a random walk in a periodic environment (RWPE).

We now briefly summarise the literature on Parrondo's games. In [9] the statedependent rule for game B is to choose Coin 2 if the player's capital is a multiple of some positive integer $M$-analysis showed the paradox could be observed for general values of $M$. In [22] it is shown that the paradox can be observed when both games A and $B$ are multiple coin games. In [23] we have the first analysis of Parrondo's games in terms of Shannon entropy and in [10] the entropy parameter spaces are graphically displayed. The probability parameter space is shown in [8]. In [15] a minimal three-state game with asymmetric transition probabilities is analysed and in [21] state-dependence on capital is replaced by dependence on the past history of the game, leading to a larger probability parameter space.

The surge of interest in analysing Parrondian games is motivated by a number of areas. Information theorists have long studied the problem of producing a fair game from biased coins [4] and the roots of this can be traced back to the work of [29]-Parrondo's games go a step further in producing a winning game from losing games. Siegman [28] has reinterpreted 'capital' of the games in terms of electron occupancies of energy levels - the paradox can then be reproduced using the rate equation approach typically used in laser analysis. In the physical world there are many types of processes where losing helps to win, such as a sacrifice in the game of chess or a valley in the fitness landscape of an animal species. Many biological effects are linked to ratchet-type phenomena and [30] have analysed enzyme transport with a four-state model. Applicability to population genetics, evolution and economics has been suggested [18]. In finance, [17] have shown that under certain conditions capital can grow by investing in an asset with negative typical growth rate. Quantum ratchets have now been experimentally realised [16] and recasting Parrondian games, based on ratchet phenomena, as quantum games $[3,5,20]$ is thus of interest.

In control theory, it can be shown that the combination of two unstable systems can become stable [1]. Velocity of propagation through an array of coupled oscillators, under certain conditions, can increase even though the damping coefficient is increased [27]. In the area of granular flow, drift can occur in a counter-intuitive direction such as is exemplified in the famous Brazil nut paradox [26]. Also declining 
branching processes can be combined to increase [12]. In [25] it is shown that noisy information can sometimes be better than clean information. In [24] it is shown that with switched diffusion processes in random media it is possible to get a positiverecurrent processes (that is, with no drift) from mixed transient processes (that is, with drifts all in the same direction) - this is almost certainly a continuous-time analogue to the Parrondian discrete-time process. Assuming we construct Parrondo's games to only deal in transactions of one unit of capital per event, then we have a skip-free process, and a statistical interpretation of the central result is that declining birth-death processes can be combined to form an increase.

In this paper we further investigate Parrondo's paradox. We construct a class of composite games and investigate their fairness by formulating the problem in the language of random walks in periodic environments (RWPE). We find many new, interesting and counter-intuitive results.

\section{Mathematical tools}

We construct a composite game from two simple games $A$ and $B$ which are modeled as RWPEs. These two games can be combined in two ways: deterministically or stochastically.

If we have played $n$ times, and $n$ is divisible by $k$ (for an integer $k$ ), we then play game $A$. If we have played $n$ times and $n$ is not divisible by $k$, we play game $B$. Thus games $A$ and $B$ are alternated in a deterministic pattern. We denote by $Y_{n}$ our capital after $n$ plays of this game.

To alternate games $A$ and $B$ randomly we toss a coin with probability $p$ of heads. If the coin comes up heads, we play game $A$, and if tails, we play game $B$. We denote by $Z_{n}$ our capital after $n$ repetitions.

The sequence of values of our capital in either of these games is a Markov random walk which changes by \pm 1 in each epoch. The two random walks $Z_{n}$ and $Y_{n}$ differ in that $Z_{n}$ is time homogeneous, while $Y_{n}$ is not time homogeneous. Moreover, $Z_{n}$ is a random walk in a periodic environment. The process $Y_{n}$ is not a RWPE, but the process $Y_{n}^{\prime} \equiv Y_{k n}$ is a RWPE.

As shown in Section 4 we extend this construction to define composite games from more than two simple games. In each case we identify the capital of the player as a RWPE.

We say that a game is fair, winning or losing if the random walk for the capital of a player, $X_{n}$, is recurrent, or transient to $\infty$ or to $-\infty$, respectively. That is, a Markov chain $X_{n}$ is

$$
\text { recurrent (fair) if } P\left\{-\infty=\lim \inf _{n \rightarrow \infty} X_{n}<\lim \sup _{n \rightarrow \infty} X_{n}=\infty\right\}=1 \text {; }
$$




$$
\begin{array}{ll}
\text { transient to } \infty \text { (winning) if } & P\left\{\lim _{n \rightarrow \infty} X_{n}=+\infty\right\}=1 ; \\
\text { transient to }-\infty \text { (losing) if } & P\left\{\lim _{n \rightarrow \infty} X_{n}=-\infty\right\}=1 .
\end{array}
$$

We note that the characterisation of a game as fair, winning, or losing by the traditional comparisons

$$
E\left[X_{n+1} \mid X_{n}\right]=X_{n}, \quad E\left[X_{n+1} \mid X_{n}\right]>X_{n} \quad \text { and } \quad E\left[X_{n+1} \mid X_{n}\right]<X_{n},
$$

respectively, does not cover the behaviour of all random walks, in particular RWPE's.

2.1. Key's criterion We consider a time homogeneous random walk, $X_{n}$, in an $N$-periodic environment, or equivalently we have a state dependent random game $\mathbf{W}$. We assume that the maximal step size in the positive direction is $R$, while in the negative direction it is $L$, that is, $P\left\{X_{n+1} \in\{-L+k, \ldots, x+R\} \mid X_{n}=k\right\}=1$. Moreover, for each $k$ the maximum right and left step sizes are always possible, that is, $P\left\{X_{n+1}=-L+k \mid X_{n}=k\right\} P\left\{X_{n+1}=R+k \mid X_{n}=k\right\}>0$. Given the environment, the walk $X_{n}$ obeys the backward master equation

$$
P\left\{\cdot \mid X_{n}=k\right\}=\sum_{j=-L}^{R} e(k, j) P\left\{\cdot \mid X_{n+1}=j+k\right\},
$$

where $e(k, j) \equiv P\left\{X_{n+1}=j+k \mid X_{n}=k\right)$ denotes the transition probability from the state $k$ to $j+k$ in one time epoch. We denote by $f_{k-i} \equiv P\left\{\cdot \mid X_{n+1}=k-i\right.$ (since $X_{n}$ is time homogeneous $f$ does not depend on $n$ ) and rewrite (2.1) as a system for the vector $\left[f_{-L+k}, f_{-L+1+k}, \ldots, f_{R-1+k}\right]^{T}$ with the matrix $\mathbf{A}_{k}$ whose entries are given by

$$
\mathbf{A}_{k}[i, j]= \begin{cases}-e(k,-L+j) / e(k,-L) & \text { if } i=1, j \neq L, \\ (1-e(k, 0)) / e(k,-L) & \text { if } i=1, j=L \\ 1 & \text { if } i \geq 2, j=i-1 \\ 0 & \text { otherwise. }\end{cases}
$$

We also define the matrix $\mathbf{M}=\mathbf{A}_{1} \mathbf{A}_{2} \cdots \mathbf{A}_{N}$.

According to [11] we define constants $d_{i}, i=1,2, \ldots, R+L$ as follows. For each eigenvalue $\lambda_{i}$ of $\mathbf{M}$ (including multiplicities), we put $d_{i}=\log \left(\left|\lambda_{i}\right|\right)$, and we list the $d_{i}$ 's in increasing order, so that $d_{1} \leq d_{2} \leq \cdots \leq d_{R+L}$. Then

if $\ln (c(\mathbf{W})) \equiv d_{R}+d_{R+1}>0$ then the RWPE $X_{n}$ is transient to $\infty$;

if $\ln (c(\mathbf{W})) \equiv d_{R}+d_{R+1}=0$ then the RWPE $X_{n}$ is recurrent;

if $\ln (c(\mathbf{W})) \equiv d_{R}+d_{R+1}<0$ then the RWPE $X_{n}$ is transient to $-\infty$.

It is shown in [13] that $X_{n}$ is recurrent if the characteristic polynomial of $\mathbf{M}$ has a double root at 1 . 


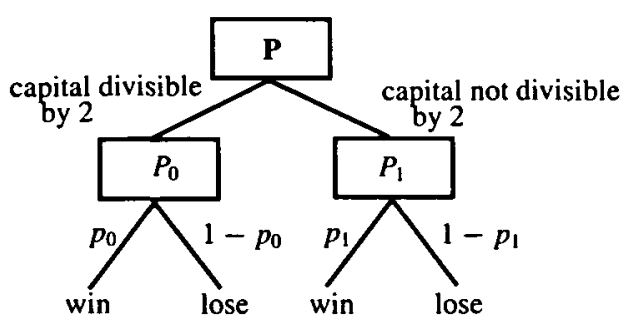

FIGURE 1. Schematic representation of a 2-(state) periodic game $\mathbf{P}=\left(P_{0}, P_{1}\right)$. Games composed of such games are discussed in Section 3.

\section{Games composed of 2-periodic games}

First we consider an example of two 2-periodic games $\mathbf{P}=\left(P_{0}, P_{1}\right)$ and $\mathbf{Q}=$ $\left(Q_{0}, Q_{1}\right)$; that is, we have two RWPE's, each of them 2-periodic (in space). If $X_{n}$ denotes the capital of a player at time $n$ who plays the game according to the rule $P$, then the transition probabilities are given by

$$
\begin{aligned}
& P\left\{X_{n+1}=x+1 \mid X_{n}=x\right\}= \begin{cases}p_{0} & \text { if } x=0 \bmod 2, \\
p_{1} & \text { if } x=1 \bmod 2,\end{cases} \\
& P\left\{X_{n+1}=x-1 \mid X_{n}=x\right\}= \begin{cases}1-p_{0} & \text { if } x=0 \bmod 2, \\
1-p_{1} & \text { if } x=1 \bmod 2 ;\end{cases}
\end{aligned}
$$

that is, if the capital is even it changes according to $P_{0}$, and if it is odd it changes according to $P_{1}$, see, for example, Figure 1 . The transition probabilities for the RWPE governed by $\mathbf{Q}$ are given by formulas analogous to (3.1), with $p^{\prime} s$ replaced by $q$ 's, with the rule $Q_{0}$ and $Q_{1}$ at the even and odd positions, respectively.

To employ the criterion of [11] to the RWPE governed by $\mathbf{P}$ we construct the matrix $\mathbf{M}$

$$
\mathbf{M}=\mathbf{A}_{1} \mathbf{A}_{0} \equiv\left[\begin{array}{cc}
1 /\left(1-p_{1}\right) & -p_{1} /\left(1-p_{1}\right) \\
1 & 0
\end{array}\right]\left[\begin{array}{cc}
1 /\left(1-p_{0}\right) & -p_{0} /\left(1-p_{0}\right) \\
1 & 0
\end{array}\right] .
$$

Since here $R=L=1, d_{R}+d_{R+1}=d_{1}+d_{2}=\log (|\operatorname{det}(\mathbf{M})|)$, so we find that the RWPE governed by $\mathbf{P}$ is transient to $-\infty$, recurrent, or transient to $+\infty$ if

$$
c(\mathbf{P}) \equiv c\left(P_{0}, P_{1}\right) \equiv \frac{p_{0} p_{1}}{\left(1-p_{0}\right)\left(1-p_{1}\right)}<(=)(>) 1 .
$$

We note that this analysis easily extends to any period $N$ of the environment, see, for example, Section 4. 
3.1. An example of a pseudo-paradox We suppose that the games $\mathbf{P}$ and $\mathbf{Q}$ are fair, that is,

$$
c\left(P_{0}, P_{1}\right)=\frac{p_{0}\left(1-p_{0}\right)}{p_{1}\left(1-p_{1}\right)}=1 \quad \text { and } \quad c\left(Q_{0}, Q_{1}\right)=\frac{q_{0}\left(1-q_{0}\right)}{q_{1}\left(1-q_{1}\right)}=1 \text {. }
$$

If we alternate (deterministically) games $\mathbf{P}$ and $\mathbf{Q}$ in that very order, then, starting at the origin, the composite game may be fair (losing) (winning) if

$$
c(\mathbf{P}, \mathbf{Q})=p_{0} q_{1} /\left(\left(1-p_{0}\right)\left(1-q_{1}\right)\right)=(<)(>) 1 .
$$

Under this strategy we play the game $P_{0}$ when the winnings are even and the game $Q_{1}$ when the winnings are odd. The games $P_{1}$ and $Q_{0}$ are never played. Hence $c(\mathbf{P}, \mathbf{Q})=$ $c\left(P_{0}, Q_{1}\right)$, and the fact that the composite game is losing when it is constructed from two winning games is just an apparent paradox. If we play the same two games when starting at an odd position then $c(\mathbf{P}, \mathbf{Q})=c\left(P_{1}, Q_{0}\right)=1 / c\left(P_{0}, Q_{1}\right)$, and we have a winning game constructed out of two winning games.

3.2. Effects of randomisation Now we construct a RWPE, $X_{n}$, by choosing at random between $\mathbf{P}$ and $\mathbf{Q}$. If our capital is even then a coin with probability of heads $g_{0}$ is used to choose whether the game $P_{0}$ or $Q_{0}$ will be played; if our capital is odd then a coin with probability of heads $g_{1}$ is used to choose between the games $P_{1}$ and $Q_{1}$. In this scheme all four games are played. The transition probabilities are given by

$$
P\left\{X_{n+1}=x+1 \mid X_{n}=x\right\}= \begin{cases}g_{0} p_{0}+\left(1-g_{0}\right) q_{0} & \text { if } x=0 \bmod 2, \\ g_{1} p_{1}+\left(1-g_{1}\right) q_{1} & \text { if } x=1 \bmod 2 .\end{cases}
$$

The fairness of the composite game is determined by the factor $c(\mathbf{P}, \mathbf{Q})$ given by

$$
c(\mathbf{P}, \mathbf{Q})=\frac{g_{0} p_{0}+\left(1-g_{0}\right) q_{0}}{\left(1-g_{0} p_{0}-\left(1-g_{0}\right) q_{0}\right)} \frac{g_{1} p_{1}+\left(1-g_{1}\right) q_{1}}{\left(1-g_{1} p_{1}-\left(1-g_{1}\right) q_{1}\right)} .
$$

By direct simplification of (3.3) we observe that if $g_{0}=g_{1}$ (and the games $\mathbf{P}$ and $\mathbf{Q}$ are fair) then the composite game is fair. Also, if $p_{1}=q_{1}$ then the composite game is fair. However, for other values of parameters two fair games can be used to compose a game which is winning, losing or fair depending on how the two simple games are randomised. Figure 2 illustrates this statement. That is, randomisation can produce Parrondo's paradox.

We also consider a game composed from two unfair games $\mathbf{P}$ and $\mathbf{Q}$. Games $\mathbf{P}$ and $\mathbf{Q}$ are taken to be unfair the same way, that is, both are losing or both are winning. We note that when one coin is used to choose whether $\mathbf{P}$ or $\mathbf{Q}$ is played, (that is, when $g_{0}=g_{1}$ in (3.2)) then the composed game is always unfair. However, if two coins are used then the randomised game may be winning when both simple games $\mathbf{P}$ and $\mathbf{Q}$ are losing. This example of Parrondo's paradox is illustrated in Figure 3. 


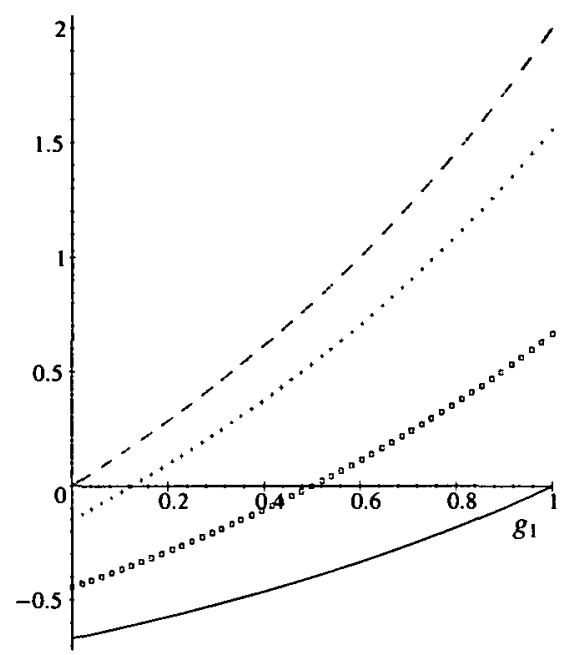

FIGURE 2. We take two 2-periodic fair games with $p_{1}=1 / 2$ and $q_{1}=1 / 4$, and plot $c(\mathbf{P}, \mathbf{Q})-1$ in (3.3) as a function of $g_{1}$ for various values of $g_{0}$; that is, the composite game is winning for positive values on the graph, and losing for negative values. We have: dashes: $g_{0}=0$; crosses: $g_{0}=1 / 8$; boxes: $g_{0}=1 / 2$; solid: $g_{0}=1$.

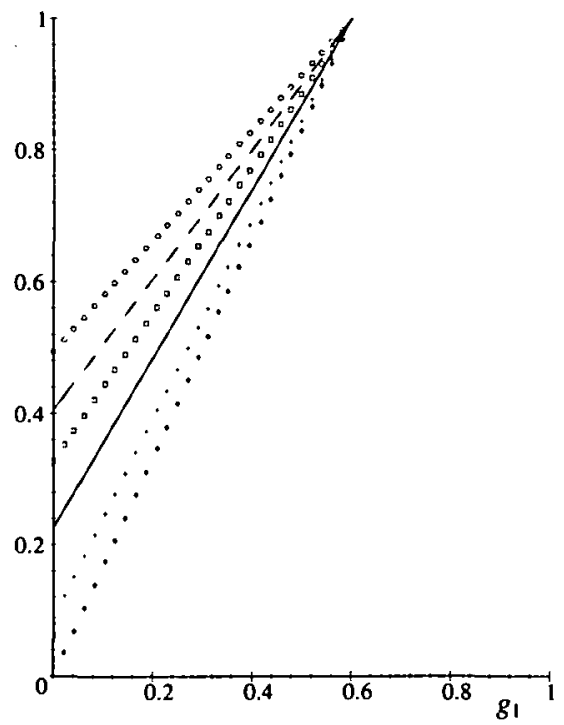

FiguRE 3. We take two 2-periodic losing games with $p_{0}=0.675, p_{1}=0.1, q_{1}=0.75$ and plot the fairness curve $c(P, Q)=1$ in (3.3) in the $\left(g_{1}, g_{0}\right)$ plane for various values of $q_{0}$ : circles: $q_{0}=0$; dashes: $q_{0}=0.075$; boxes: $q_{0}=0.125$; solid: $q_{0}=0.175$; crosses: $q_{0}=0.225$; diamonds: $q_{0}=0.25$. The composite game is winning when $g_{0}$ and $g_{1}$ are selected above the fairness curve. 
3.3. Three 2-periodic games We consider three 2-periodic games $\mathbf{P}, \mathbf{Q}$ and $\mathbf{R}$, each defined analogously to (3.1), with transition probabilities given in terms of $p_{0}, p_{1}, q_{0}, q_{1}$, and $r_{0}, r_{1}$, respectively. We construct the composite game $\mathbf{P Q R}$. We investigate whether the composite game may be winning (losing) if the individual games $\mathbf{P}, \mathbf{Q}$ and $\mathbf{R}$ are fair. If the capital of the player at time $n$ is $X_{n}$ then the capital at time $3 n, Y_{n} \equiv X_{3 n}$, is a random walk in a 2-periodic environment, taking steps of $\pm 3, \pm 1$. Specifically, we have

$$
\begin{aligned}
& P\left\{Y_{n+1}=x+3 \mid Y_{n}=x\right\}=\left\{\begin{array}{l}
a_{0} \equiv p_{0} q_{1} r_{0} \quad \text { if } x=0 \bmod 2, \\
a_{1} \equiv p_{1} q_{0} r_{1} \text { if } x=1 \bmod 2,
\end{array}\right. \\
& P\left\{Y_{n+1}=x-3 \mid Y_{n}=x\right\}=\left\{\begin{array}{l}
b_{0} \equiv\left(1-p_{0}\right)\left(1-q_{1}\right)\left(1-r_{0}\right) \text { if } x=0 \bmod 2, \\
b_{1} \equiv\left(1-p_{1}\right)\left(1-q_{0}\right)\left(1-r_{1}\right) \text { if } x=1 \bmod 2,
\end{array}\right. \\
& P\left\{Y_{n+1}=x+1 \mid Y_{n}=x\right\}=\left\{\begin{array}{cc}
c_{0} \equiv p_{0} q_{1}\left(1-r_{0}\right)+p_{0}\left(1-q_{1}\right) r_{0} \\
+\left(1-p_{0}\right) q_{1} r_{0} & \text { if } x=0 \bmod 2, \\
c_{1} \equiv p_{1} q_{0}\left(1-r_{1}\right)+p_{1}\left(1-q_{0}\right) r_{1} \\
+\left(1-p_{1}\right) q_{0} r_{1} & \text { if } x=1 \bmod 2,
\end{array}\right. \\
& P\left\{Y_{n+1}=x-1 \mid Y_{n}=x\right\}=\left\{\begin{array}{cc}
d_{0} \equiv\left(1-p_{0}\right)\left(1-q_{1}\right) r_{0}+\left(1-p_{0}\right) q_{1}\left(1-r_{0}\right) \\
+p_{0}\left(1-q_{1}\right)\left(1-r_{0}\right) & \text { if } x=0 \bmod 2, \\
d_{1} \equiv\left(1-p_{1}\right)\left(1-q_{0}\right) r_{1}+\left(1-p_{1}\right) q_{0}\left(1-r_{1}\right) \\
+p_{1}\left(1-q_{0}\right)\left(1-r_{1}\right) & \text { if } x=1 \bmod 2 .
\end{array}\right.
\end{aligned}
$$

Moreover, the random walk $Z_{n} \equiv Y_{2 n}$ is an ordinary random walk, that is, a sum of i.i.d. random variables taking values $\pm 6, \pm 4, \pm 2$, and 0 , and the transition probabilities are

$$
\begin{aligned}
P\left\{Z_{n+1}=z+6 \mid Z_{n}=z\right\} & =a_{0} a_{1}, \\
P\left\{Z_{n+1}=z-6 \mid Z_{n}=z\right\} & =b_{0} b_{1}, \\
P\left\{Z_{n+1}=z+4 \mid Z_{n}=z\right\} & =a_{0} c_{1}+c_{0} a_{1}, \\
P\left\{Z_{n+1}=z-4 \mid Z_{n}=z\right\} & =b_{0} d_{1}+d_{0} b_{1}, \\
P\left\{Z_{n+1}=z+2 \mid Z_{n}=z\right\} & =c_{0} c_{1}+a_{0} d_{1}+a_{1} d_{0}, \\
P\left\{Z_{n+1}=z-2 \mid Z_{n}=z\right\} & =d_{0} d_{1}+b_{0} c_{1}+b_{1} c_{0}, \\
P\left\{Z_{n+1}=z \mid Z_{n}=z\right\} & =d_{0} c_{1}+d_{1} c_{0}+a_{0} b_{1}+a_{1} b_{0} .
\end{aligned}
$$

If the games $\mathbf{P}, \mathbf{Q}$ and $\mathbf{R}$ are fair, then by direct calculations, $E Z_{n}=0$, so the composite game is fair, and there is no paradox in this case. 


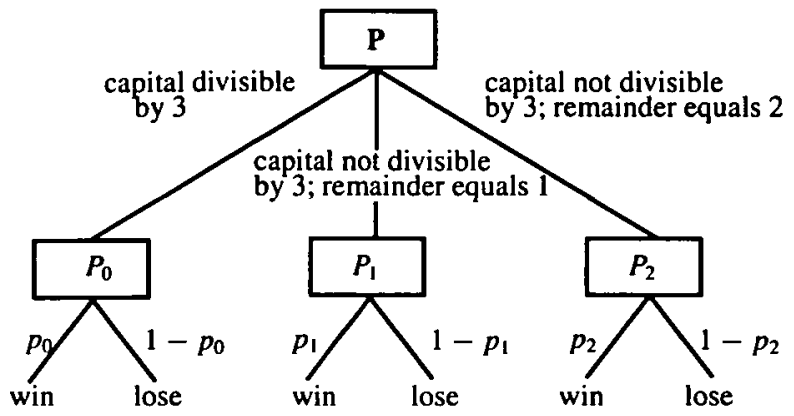

FIGURE 4. Schematic representation of a 3-(state) periodic game $P=\left(P_{0}, P_{1}, P_{2}\right)$. Games composed of such games are discussed in Section 4.

\section{Games composed of 3-periodic games}

We consider games composed of 3-periodic games $\mathbf{P}, \mathbf{Q}$, and $\mathbf{R}$. We define a 3-periodic game $\mathbf{P}=\left(P_{0}, P_{1}, P_{2}\right)$ by its transition probabilities as

$$
P\left\{X_{n+1}=x+1 \mid X_{n}=x\right\}= \begin{cases}p_{0} & \text { if } x=0 \bmod 3, \\ p_{1} & \text { if } x=1 \bmod 3, \\ p_{2} & \text { if } x=2 \bmod 3,\end{cases}
$$

compare Figure 4. Transition probabilities of the games $\mathbf{Q}$ and $\mathbf{R}$ are defined by formulas analogous to (4.1) with $p$ 's replaced by $q$ 's and $r$ 's, respectively. According to [11], to determine the fairness of the game $\mathbf{P}$ we consider the product of the eigenvalues of the matrix $\mathbf{M} \equiv \mathbf{A}_{1} \mathbf{A}_{2} \mathbf{A}_{0}$ where

$$
\mathbf{A}_{i}=\left[\begin{array}{cc}
1 /\left(1-p_{i}\right) & -p_{i} /\left(1-p_{i}\right) \\
1 & 0
\end{array}\right] \quad i=0,1,2
$$

If $p_{0} p_{1} p_{2} /\left[\left(1-p_{0}\right)\left(1-p_{1}\right)\left(1-p_{2}\right)\right]=(<)(>) 1$ then the game $\mathbf{P}$ is fair (losing) (winning).

4.1. Two 3-periodic games To analyse a game $(P, Q)$, where $P$ and $Q$ are 3-periodic games, we construct a random walk $Y_{n} \equiv X_{2 n}$ where $X_{n}$ denotes the position of the walker at time $n$. The transition probabilities for $Y_{n}$ are given by

$$
P\left\{Y_{n+1}=x+2 \mid Y_{n}=x\right\}= \begin{cases}a_{0} \equiv p_{0} q_{1} & \text { if } x=0 \bmod 3, \\ a_{1} \equiv p_{1} q_{2} & \text { if } x=1 \bmod 3, \\ a_{2} \equiv p_{2} q_{0} & \text { if } x=2 \bmod 3,\end{cases}
$$




$$
\begin{gathered}
P\left\{Y_{n+1}=x \mid Y_{n}=x\right\}= \begin{cases}b_{0} \equiv p_{0}\left(1-q_{1}\right)+\left(1-p_{0}\right) q_{2} & \text { if } x=0 \bmod 3, \\
b_{1} \equiv p_{1}\left(1-q_{2}\right)+\left(1-p_{1}\right) q_{0} & \text { if } x=1 \bmod 3, \\
b_{2} \equiv p_{2}\left(1-q_{0}\right)+\left(1-p_{2}\right) q_{1} & \text { if } x=2 \bmod 3,\end{cases} \\
P\left\{Y_{n+1}=x-2 \mid Y_{n}=x\right\}= \begin{cases}c_{0} \equiv\left(1-p_{0}\right)\left(1-q_{2}\right) & \text { if } x=0 \bmod 3, \\
c_{1} \equiv\left(1-p_{1}\left(1-q_{0}\right)\right. & \text { if } x=1 \bmod 3, \\
c_{2} \equiv\left(1-p_{2}\right)\left(1-q_{1}\right) & \text { if } x=2 \bmod 3 .\end{cases}
\end{gathered}
$$

Hence $Y_{n}$ is a 3-periodic random walk taking steps \pm 2 and zero. To employ Key's criterion most efficiently we observe that it is sufficient to analyse $Y_{n}$ as a RWPE on the even integers which visits only nearest neighbours. To this end we construct the $2 \times 2$ matrix $\mathbf{M}=\mathbf{A}_{1} \mathbf{A}_{2} \mathbf{A}_{0}$ where

$$
\mathbf{A}_{i}=\left[\begin{array}{cc}
\left(1-b_{i}\right) / c_{i} & -a_{i} / c_{i} \\
1 & 0
\end{array}\right] \quad i=0,1,2
$$

The determinant of $\mathbf{M}$ is given by

$$
\operatorname{det}(\mathbf{M})=\frac{p_{0} p_{1} p_{2} q_{0} q_{1} q_{2}}{\left(1-p_{0}\right)\left(1-p_{1}\right)\left(1-p_{2}\right)\left(1-q_{0}\right)\left(1-q_{1}\right)\left(1-q_{2}\right)} .
$$

Hence if the games $\mathbf{P}$ and $\mathbf{Q}$ are fair, the composite game is fair and no paradox is observed.

We note that this reduction in the dimension of $\mathbf{M}$ occurs whenever the temporal period is even, since the step sizes of the derived process $Y_{n}$ are even. Specifically, if the temporal period is $T$ is even then $\mathbf{M}$ can be taken to be the product of $T \times T$ matrices, and when the temporal period $T$ is odd, then $\mathbf{M}$ is the product of $2 T \times 2 T$ matrices.

4.2. Two 3-periodic games randomised We construct a composite game from two 3-periodic random games $\mathbf{P}=\left(P_{0}, P_{1}, P_{2}\right)$ and $\mathbf{Q}=\left(Q_{0}, Q_{1}, Q_{2}\right)$ by selecting at random the game to be played at each step, with probability of playing $P_{i}$ equal to $g_{i}$, $i=0,1,2$. That is, we define a 3 -periodic random walk $Y_{n}$ taking values \pm 1 with transition probabilities

$$
P\left\{Y_{n+1}=x+1 \mid Y_{n}=x\right\}= \begin{cases}\rho_{0} \equiv g_{0} p_{0}+\left(1-g_{0}\right) q_{0} & \text { if } x=0 \bmod 3, \\ \rho_{1} \equiv g_{1} p_{1}+\left(1-g_{1}\right) q_{1} & \text { if } x=1 \bmod 3, \\ \rho_{2} \equiv g_{2} p_{2}+\left(1-g_{2}\right) q_{2} & \text { if } x=2 \bmod 3 .\end{cases}
$$

The walk $Y_{n}$ is recurrent (transient to $\infty$ ) (transient to $-\infty$ ) according to

$$
c(\mathbf{P}, \mathbf{Q}) \equiv \frac{\rho_{0} \rho_{1} \rho_{2}}{\left(1-\rho_{0}\right)\left(1-\rho_{1}\right)\left(1-\rho_{2}\right)}=(>)(<) 1 .
$$




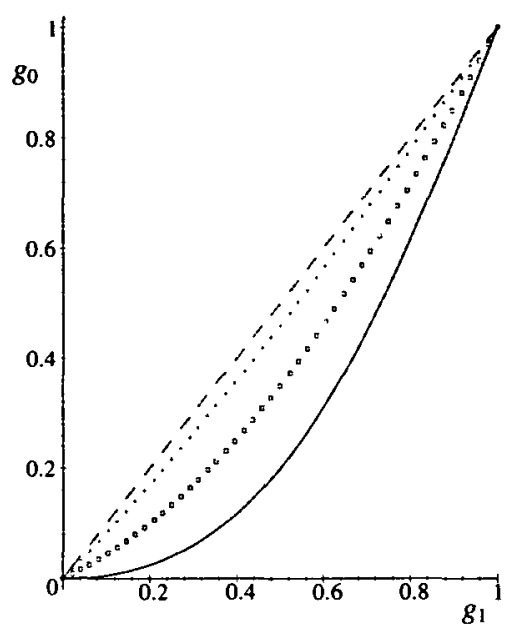

FIGURE 5. We take two fair 3-periodic games with $g_{1}=g_{2}, p_{1}=p_{2}=1 / 2$, and $q_{1}=q_{2}$. For a fixed value of $q_{1}$ we plot curves along which the composite game is fair, in the space $\left(g_{1}, g_{0}\right)$. As $q_{1}$ varies from 0 to $1 / 2$ the composite game is losing above and winning below its corresponding "fairness" curve; solid: $q_{1}=0$; boxes: $q_{1}=1 / 6$; crosses: $q_{1}=1 / 3$; dashes: the line $g_{0}=g_{1}$. As $q_{1}$ varies from $1 / 2$ to 1 the composite game is winning below and losing above its corresponding "fairness" curve. In this case the problem is symmetric, so that we have: solid: $q_{1}=1$; boxes $q_{1}=5 / 6$; crosses: $q_{1}=2 / 3$.

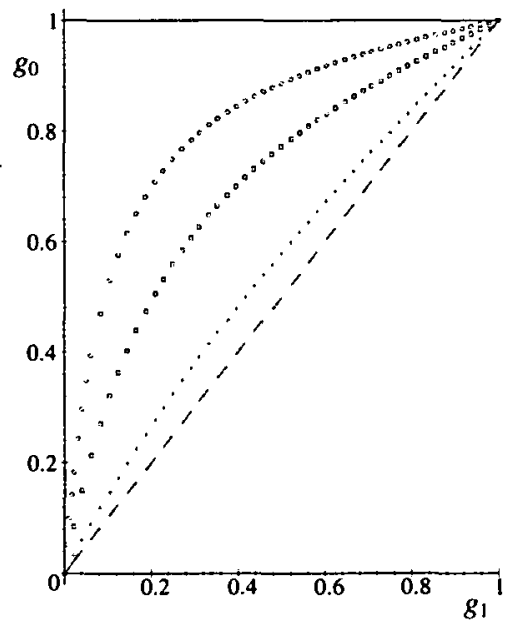

FlGURE 6. We take two fair 3-periodic games with $g_{1}=g_{2}, p_{1}=p_{2}=1 / 2$, and $q_{0}=q_{2}$. We plot the curves along which the composite game is fair in the space $\left(g_{1}, g_{0}\right)$, for a fixed value of $q_{0}$. As $q_{0}$ varies from 0 to $1 / 2$, the game is winning above and losing below each of its "fairness" curves; solid: $q_{0}=0$; circles: $q_{0}=1 / 12$; boxes: $q_{0}=1 / 6$; crosses: $q_{0}=1 / 3$; dashes: the line $g_{0}=g_{1}$. As $q_{0}$ increases from $1 / 2$ to 1 the game is losing above and winning below its fairness curve. The case of this example is symmetric so that we have: crosses: $q_{0}=2 / 3$; boxes: $q_{0}=5 / 6$; circles: $q_{0}=11 / 12$. 
We investigate the value of $c(\mathbf{P}, \mathbf{Q})$ for various values of the parameters. We note that if the games $\mathbf{P}$ and $\mathbf{Q}$ are fair with $p_{1}=p_{2}=q_{1}=q_{2}$ then the randomised composite game is fair for all possible choices of $g_{i}$ 's. Next we take two fair games with fixed values $p_{1}=p_{2}$ and $q_{1}=q_{2}$, that is, $\mathbf{P}=\left(P_{0}, P_{1}, P_{1}\right)$ and $\mathbf{Q}=\left(Q_{0}, Q_{1}, Q_{1}\right)$.

We investigate how randomisation affects the fairness of the composite game. As shown in Figure 5 there is a wide range of parameters for which the randomised game is unfair, so that randomisation induces the paradox. We note that the range of the randomisation parameters $g^{\prime} s$ is affected by the way games $\mathbf{P}$ and $\mathbf{Q}$ are structured. In the examples illustrated in Figure 6 we take two fair games $\mathbf{P}=\left(P_{0}, P_{1}, P_{1}\right)$ and $\mathbf{Q}=\left(Q_{0}, Q_{1}, Q_{0}\right)$ and we note that the range of parameters $g_{0}, g_{1}=g_{2}$ for which the randomised game composed from these "shifted" games is larger compared to the previous "not-shifted" case, see, for example, Figures 5 and 6.

4.3. Three 3-periodic games First we construct a game (PQR) in which the 3-periodic games $\mathbf{P}, \mathbf{Q}$ and $\mathbf{R}$ are played in a deterministic order. We show that if all three games are fair then the resulting game may be losing. We also show that if all three games are winning then the composite game may be losing. If $X_{n}$ denotes the winnings of the composite game at time $n$ then its transition probabilities are given by: if $x=0 \bmod 3$ then

$$
\begin{aligned}
P\left\{X_{n+3}=x+3 \mid X_{n}=x\right\}=a_{0} \equiv & p_{0} q_{1} r_{2}, \\
P\left\{X_{n+3}=x-3 \mid X_{n}=x\right\}=b_{0} \equiv & \left(1-p_{0}\right)\left(1-q_{2}\right)\left(1-r_{1}\right), \\
P\left\{X_{n+3}=x+1 \mid X_{n}=x\right\}=c_{0} \equiv & p_{0}\left(1-q_{2}\right) r_{1} \\
& +\left(1-p_{0}\right) q_{2}\left(1-r_{0}\right)+p_{0}\left(1-q_{1}\right)\left(1-r_{0}\right), \\
P\left\{X_{n+3}=x-1 \mid X_{n}=x\right\}=d_{0} \equiv & \left(1-p_{0}\right)\left(1-q_{2}\right) r_{1} \\
& +\left(1-p_{0}\right) q_{2}\left(1-r_{0}\right)+p_{0}\left(1-q_{1}\right)\left(1-r_{0}\right) ;
\end{aligned}
$$

if $x=1 \bmod 3$ then

$$
\begin{aligned}
P\left\{X_{n+3}=x+3 \mid X_{n}=x\right\}=a_{1} \equiv & p_{1} q_{2} r_{0}, \\
P\left\{X_{n+3}=x-3 \mid X_{n}=x\right\}=b_{1} \equiv & \left(1-p_{1}\right)\left(1-q_{0}\right)\left(1-r_{2}\right), \\
P\left\{X_{n+3}=x+1 \mid X_{n}=x\right\}=c_{1} \equiv & p_{1} q_{2}\left(1-r_{0}\right)+p_{1}\left(1-q_{2}\right) r_{1}+\left(1-p_{1}\right) q_{0} r_{1}, \\
P\left\{X_{n+3}=x-1 \mid X_{n}=x\right\}=d_{1} \equiv & \left(1-p_{1}\right)\left(1-q_{0}\right) r_{2} \\
& +\left(1-p_{1}\right) q_{0}\left(1-r_{1}\right)+p_{1}\left(1-q_{2}\right)\left(1-r_{1}\right) ;
\end{aligned}
$$

if $x=2 \bmod 3$ then

$$
\begin{aligned}
P\left\{X_{n+3}=x+3 \mid X_{n}=x\right\} & =p_{2} q_{0} r_{1} \equiv a_{2}, \\
P\left\{X_{n+3}=x-3 \mid X_{n}=x\right\}= & \left(1-p_{2}\right)\left(1-q_{1}\right)\left(1-r_{0}\right) \equiv b_{2}, \\
P\left\{X_{n+3}=x+1 \mid X_{n}=x\right\}= & p_{2} q_{0}\left(1-r_{1}\right)+p_{2}\left(1-q_{0}\right) r_{2}+\left(1-p_{2}\right) q_{1} r_{2} \equiv c_{2}, \\
P\left\{X_{n+3}=x-1 \mid X_{n}=x\right\}= & d_{2} \equiv\left(1-p_{2}\right)\left(1-q_{1}\right) r_{0} \\
& +\left(1-p_{2}\right) q_{1}\left(1-r_{2}\right)+p_{2}\left(1-q_{0}\right)\left(1-r_{2}\right) .
\end{aligned}
$$


Hence we define the walk in a periodic environment by $Y_{n} \equiv X_{3 n}$, and determine its transient/recurrent properties by investigating locations of the eigenvalues of the matrix $\mathbf{M}=\mathbf{A}_{1} \mathbf{A}_{2} \mathbf{A}_{0}$. Since the walk $Y_{n}$ takes on the values $\pm 3, \pm 1, R=L=3$, each of the matrices $\mathbf{A}_{i}, i=1,2,3$, is a $6 \times 6$ matrix given by

$$
\mathbf{A}_{i}=\left[\begin{array}{cccccc}
0 & -d_{i} / b_{i} & 1 / b_{i} & -c_{i} / b_{i} & 0 & -a_{i} / b_{i} \\
1 & 0 & 0 & 0 & 0 & 0 \\
0 & 1 & 0 & 0 & 0 & 0 \\
0 & 0 & 1 & 0 & 0 & 0 \\
0 & 0 & 0 & 1 & 0 & 0 \\
0 & 0 & 0 & 0 & 1 & 0
\end{array}\right], \quad i=0,1,2
$$

The examples illustrated in Figure 7 and 8 show that the game composed deterministically from fair (non-symmetric) games can be fair or losing or winning, depending on parameters of the problem. This form of the paradox is not observed when composing (deterministically) two or three 2-periodic games. We observe even more interesting behaviour when composing deterministically unfair (losing) games. Figures 9 and 10 show such examples.

By varying just one parameter, it is possible to change the composed game from losing to winning, change it back to losing, and then back again to winning, see, for example, Figure 9. Three state transitions of the composed game have not been observed before.

\section{Conclusions}

Given the highly nonlinear nature of the recurrence/transience criteria for random walks in periodic environments as a function of the process parameters, it is not surprising that any scheme that combines recurrent random walks in periodic environment to produce new random walks in a periodic environments will produce transient processes, except in trivial cases.

What is surprising to us is that one can find non-trivial cases where random walks in periodic environments with negative drift can be combined to form random walks in periodic environments with positive drift, Parrondo's paradox. We believe that these reversals arise because combining these processes in the manner described above radically changes the frequency at which transitions are governed by probability distributions with positive means, and that future investigations should center on investigating these frequencies. It is likely that it will be easier to understand this phenomenon in the case of stochastic combinations of the processes since these always lead to the study of nearest neighbour random walks in periodic environments.

When a composite game is constructed from simple 3-periodic games, Parrondo's paradox is much more interesting and complex than in the case of 2-periodic games. As 


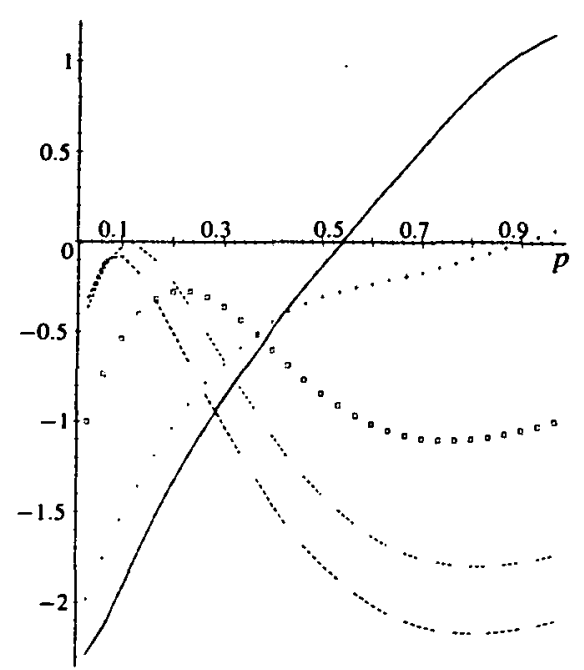

FIGURE 7. We take 3 fair games with $p_{0}=p_{1} \equiv p, q_{0}=q_{1} \equiv q$, and $r_{0}=r_{1} \equiv r$, and plot $\ln (c(\mathbf{P}, \mathbf{Q}, \mathbf{R}))=d_{3}+d_{4}$, compare (2.2), as a function of $p$, for $g=0.1$ and various values of $r$, solid: $r=3 / 4$; crosses: $r=1 / 2$, boxes: $r=1 / 4$; short dashes: $r=1 / 8$; long dashes: $r=1 / 16$. We note that the composite game is always losing, no matter what $p$ is, for a wide range of values of the parameter $r$. If $\ln (c(\mathbf{P}, \mathbf{Q}, \mathbf{R}))$ is positive (zero) (negative) then the game $(\mathbf{P}, \mathbf{Q}, \mathbf{R})$ ) is winning (fair) (losing).

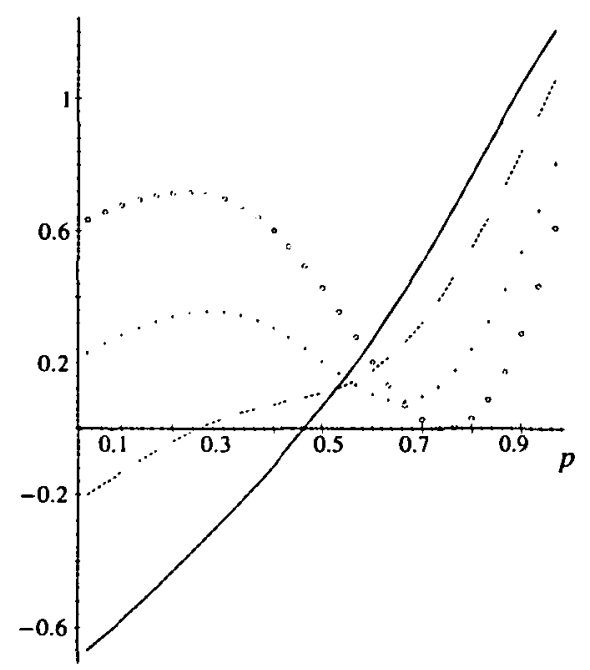

FIGURE 8. We take 3 fair games with $p_{0}=p_{1} \equiv p, q_{0}=q_{1} \equiv q$, and $r_{0}=r_{1} \equiv r$, and plot $\ln (c(\mathbf{P}, \mathbf{Q}, \mathbf{R}))=d_{3}+d_{4}$, compare (2.2), as a function of $p$, for $q=3 / 4$ and various values of $r$ : circles: $r=3 / 4$, crosses: $r=5 / 8$; dashes: $r=1 / 2$; solid: $r=3 / 8$. If $\ln (c(P, Q, R)$ ) is positive (zero) (negative) then the game (P, $\mathbf{Q}, \mathbf{R})$ ) is winning (fair) (losing). 


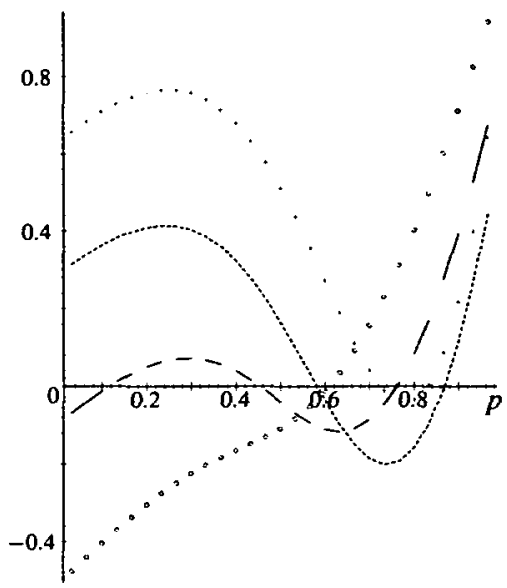

FIGURE 9. We take 3 losing games with $p_{0}=p_{1} \equiv p, q_{0}=q_{1} \equiv q$, and $r_{0}=r_{1} \equiv r$, and $p_{2}=0.8(1-p)^{2} /\left((1-p)^{2}+p^{2}\right), q_{2}=0.8(1-q)^{2} /\left((1-q)^{2}+q^{2}\right)$, and $r_{2}=0.9(1-r)^{2} /\left((1-r)^{2}+r^{2}\right)$. We set $q=3 / 4$, so that $q_{2}=0.08$, and plot $\ln (c(\mathbf{P}, \mathbf{Q}, \mathbf{R}))=d_{3}+d_{4}$, compare (2.2), as a function of $p$ for various values of $r$, circles: $r=1 / 2,\left(r_{2}=0.45\right)$; solid: $r=5 / 8,\left(r_{2}=0.238235 \ldots\right)$; dashes: $r=3 / 4,\left(r_{2}=0.09\right)$; crosses $r=7 / 8,\left(r_{2}=0.018\right)$. If $\ln (c(\mathbf{P}, \mathbf{Q}, \mathbf{R}))$ is positive (zero) (negative) then the game ( $\mathbf{P}, \mathbf{Q}, \mathbf{R})$ ) is winning (fair) (losing).

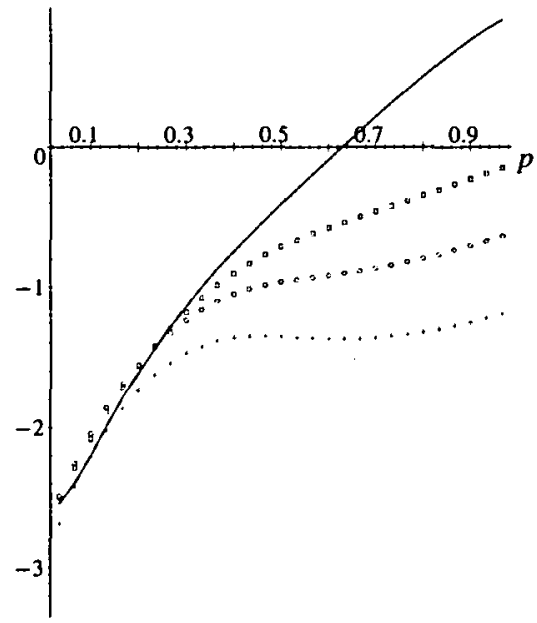

FIGURE 10. We take 3 losing games with $p_{0}=p_{1} \equiv p, q_{0}=q_{1} \equiv q$, and $r_{0}=r_{1} \equiv r$, and $p_{2}=0.8(1-p)^{2} /\left((1-p)^{2}+p^{2}\right), q_{2}=0.8(1-q)^{2} /\left((1-q)^{2}+q^{2}\right)$, and $r_{2}=0.9(1-r)^{2} /\left((1-r)^{2}+r^{2}\right)$. We set $q=0.1$ so that $q_{2}=0.8890 \ldots$, and plot $\ln (c(P, Q, \mathbf{R}))=d_{3}+d_{4}$, compare (2.2), as a function of $p$ for various values of $r$, solid: $r=3 / 4,\left(r_{2}=0.9\right)$; box: $r=1 / 2,\left(r_{2}=0.45\right)$; circles: $r=3 / 8$, $\left(r_{2}=0.66176 \ldots\right)$; crosses $r=1 / 4,\left(r_{2}=0.81\right)$. If $\ln (c(P, Q, R))$ is positive (zero) (negative) then the game $(\mathbf{P}, \mathbf{Q}, \mathbf{R})$ ) is winning (fair) (losing). 
shown in Figure 9, by varying one parameter three-state transitions of the composed game may be observed. This phenomenon seems to be similar to changes of the direction of the current in a multiplicative stochastic ratchet as observed in [14].

As shown in Section 3, a game constructed by composing deterministically 2-periodic fair games leads to a pseudo-paradox. In general, a game composed from an even number of 2-periodic fair games always exhibits a pseudo-paradox [13]. That is, if each simple game is 2-state periodic with an even temporal period, then the composite game is constructed only from half of the simple games, and the composite game may be winning, or losing, or fair. Moreover, when each of the simple games is fair with an odd temporal period, and the composite game is composed deterministically, then Parrondo's paradox never occurs. An example in Section 3 of a game composed from 2-state periodic and 3-temporal periodic games illustrates this statement.

\section{Acknowledgements}

D.A. would like to acknowledge funding from the Sir Ross and Sir Keith Smith Fund, GTECH, the Australian Research Council and SA Lotteries. Also thanks are due to Prof. A. E. Siegman, Stanford University, USA and Prof. J. Maynard Smith, University of Sussex, UK for useful communications. The 'Boston Interpretation' originated during a round table discussion with Prof. H. E. Stanley's group at Boston University.

\section{References}

[1] A. Allison and D. Abbott, "Stochastically switched control systems", in Proc. 2nd Int. Conf. on Unsolved Problems of Noise (UPoN'99), Adelaide, Australia, 11-15th July 1999 (eds. D. Abbott and L. B. Kish), (American Institute of Physics Conference Proceedings 511, 2000) 249-254.

[2] C. R. Doering, "Randomly rattled ratchets", Nuovo Cimento 17D (1995) 685-679.

[3] J. Eisert, M. Wilkens and M. Lewenstein, "Quantum games and quantum strategies", Phys. Rev. Lett. 83 (1999) 3077-3080.

[4] L. Gargamo and U. Vaccaro, "Efficient generation of fair dice with few biased coins", IEEE Trans. Info. Theory. 45 (1999) 1600-1606.

[5] L. Goldenberg, L. Vaidman and S. Wiesner, "Quantum gambling", Phys. Rev. Lett. 82 (1999) 3356-3359.

[6] G. P. Harmer and D. Abbott, "Losing strategies can win by Parrondo's paradox", Nature 402 (1999) 846.

[7] G. P. Harmer and D. Abbott, “Parrondo's paradox". Statistical Science 14 (1999) 206-213.

[8] G. P. Harmer, D. Abbott and P. Taylor, "The paradox of Parrondo's games", Proc. R. Soc. Lond. A. $456(2000) 247-259$. 
[9] G. P. Harmer, D. Abbott, P. Taylor and J. M. R. Parrondo, "Parrondo's paradoxical games and the discrete Brownian ratchet", in Proc. 2nd Int. Conf. on Unsolved Problems of Noise (UPoN'99), Adelaide, Australia, 11-15th July 1999 (eds. D. Abbott and L. B. Kish), (American Institute of Physics Conference Proceedings 511, 2000) 189-200.

[10] G. P. Harmer, D. Abbott, P. Taylor, C. E. M. Pearce and J. M. R. Parrondo, "Information entropy and Parrondo's discrete-time ratchet", in Proc. Stochaos, Ambleside, UK, 16-20 August 1999 (eds. D. S. Broomhead, E. A. Luchinskaya, P. V. E. McClintock and T. Mullin), (American Institute of Physics Conference Proceedings 502, 2000) 544-549.

[11] E. S. Key, "Recurrence and transience criteria for random walk in a random environment", Ann. Prob. 12 (1984) 529-560.

[12] E. S. Key, "Computable examples of the maximal Lyapunov exponent", Probab. Th. Rel. Fields 75 (1987) 97-107.

[13] E. S. Key and H. Volkmer, "Eigenvalue multiplicities of products of companion matrices", Electronic J. Lin. Algebra 11 (2004) 396-409.

[14] M. M. Klosek and R. W. Cox, "Steady-state currents in sharp stochastic ratchets", in Proc. Stochaos, Ambleside, UK, 16-20 August 1999 (eds. D. S. Broomhead, E. A. Luchinskaya, P. V. E. McClintock and T. Mullin), (American Institute of Physics Conference Proceedings 502, 2000) 325-330.

[15] Y. Lee, D. Abbott and H. E. Stanley, "Minimal Parrondian ratchet: an exactly solvable model", Phys. Rev. Lett. 91 (2003) 220601.

[16] H. Linke, T. E. Humphrey, A. Löfgren, A. O. Sushkov, R. Newbury, R. P. Taylor and P. Omling, "Experimental tunneling ratchets", Science 286 (1999) 2314-2317.

[17] S. Maslov and Y. Zhang, "Optimal investment strategy for risky assets", Int. J. Theor. Appl. Fin. 1 (1998) 377-387.

[18] P. V. E. McClintock, “Unsolved problems of noise”, Nature 401 (1999) 23-25.

[19] J. Maynard Smith, "personal communication", 1999.

[20] D. A. Meyer, "Quantum strategies", Phys. Rev. Lett. 82 (1998) 1052-1055.

[21] J. M. R. Parrondo, G. P. Harmer and D. Abbott, "New paradoxical games based on Brownian ratchets", Phys. Rev. Lett. 85 (2000) 5226-5229.

[22] C. E. M. Pearce, "On Parrondo's paradoxical games", in Proc. 2nd Int. Conf. on Unsolved Problems of Noise (UPoN'99), Adelaide, Australia, 11-15th July 1999 (eds. D. Abbott and L. B. Kish), (American Institute of Physics Conference Proceedings 511, 2000) 201-206.

[23] C. E. M. Pearce, "Entropy, Markov information sources and Parrondo games", in Proc. 2nd Int. Conf. on Unsolved Problems of Noise (UPoN'99), Adelaide, Australia, 11-15th July 1999 (eds. D. Abbott and L. B. Kish), (American Institute of Physics Conference Proceedings 511, 2000) 207-212.

[24] R. Pinsky and M. Scheutzow, "Some remarks and examples concerning transience and recurrence of random diffusions", Ann. Inst. H. Poincaré Probab. Statist. 28 (1992) 519-536.

[25] L. Plaskota, "How to benefit from noise", J. Complexity 12 (1996) 175-184.

[26] A. Rosato, K. J. Strandburg, F. Prinz and R. H. Swendsen, "Why the Brazil nuts are on top: size segregation of particulate matter shaking", Phys. Rev. Lett. 58 (1987) 1038-1040.

[27] A. Sarmiento, R. Reigada, A. H. Romero and K. Lindenberg, "Enhanced pulse propagation in non-linear arrays of oscillators", Phys. Rev. E 60 (1999) 5317-5326.

[28] A. E. Seigman, "personal communication", 1999.

[29] J. von Neumann, "Various techniques used in connection with random digits, notes by G. E. Forsythe, National Bureau of Standards". Appl. Math. Ser. 12 (1951) 36-38.

[30] H. V. Westerhoff, T. Y. Tsong, P. B. Chock, Y. Chen and R. D. Astumian, "How enzymes can capture and transmit fee energy from an oscillating electric field", Proc. Natl. Acad. Sci. 83 (1986) $4734-4738$. 